\title{
El maltrato infantil: un problema mundial
}

\author{
Rosalinda Santana-Tavira, M.C., ${ }^{(I)}$ Roberto Sánchez-Ahedo, M.C., M. en E.M., ${ }^{(1)}$ \\ Emilio Herrera-Basto, M.C. ${ }^{(2)}$
}

\section{Santana-Tavira R, Sánchez-Ahedo R, Herrera-Basto E. El maltrato infantil: un problema mundial. Salud Publica Mex 1998;40:58-65.}

\section{Resumen}

Al abordar el maltrato infantil se presentan diversos problemas: desconocimiento de la verdadera proporción de dicha problemática; raíces culturales e históricas profundas; diversidad de opiniones en cuanto a su definición y clasificación; dificultades en la investigación y, finalmente, una diversidad de consideraciones sobre sus repercusiones y su manejo terapéutico. En el presente artículo se estudia el maltrato infantil desde sus antecedentes históricos, así como sus clasificaciones, sus definiciones y su epidemiología. Asimismo, se revisan las repercusiones y se plantean las alternativas de tratamiento que en la bibliografía existente se han manejado como fundamentales para enfrentar este fenómeno cada vez más alarmante. Queda subrayada la necesidad de unificar criterios en cuanto a la definición y clasificación de información científica en torno a datos demográficos que, finalmente, hablarán de la realidad del problema, de los avances relacionados con sus causas, su diagnóstico, sus medidas preventivas y su tratamiento. Es fundamental utilizar medidas tendientes a prevenir el maltrato, pues una gran parte de los problemas en el niño se ven reflejados en la vida adulta. Se comparan las distintas clasificaciones en torno al tema, así como las características tanto del agredido como del agresor en los distintos tipos de maltrato.

Palabras clave: maltrato a los niños; abuso sexual infantil; México

\section{Santana-Tavira R, Sánchez-Ahedo R, Herrera-Basto $E$. \\ Child abuse: A worldwide problem. Salud Publica Mex 1 998;40:58-65.}

\section{Abstract}

Several problems are encountered in the study of child abuse: ignorance of its real proportions, deep cultural and historical roots, diversity of opinion as to its definition and classification and, finally, very diverse considerations on its repercussions and therapeutic management. The present study approaches child abuse from its historical precedents, its classifications, definitions and epidemiology. In addition, repercussions are reviewed, and treatment alternatives considered which are held as fundamental to confront this alarmingly increasing phenomenon. It is important to unify criteria as to the definition and classification of scientific information surrounding demographic data which, in the end, will situate the problem, the progress related to its causes, diagnosis, preventive measures and treatment. It is extremely important to prevent child abuse by all possible means, since this harm is reflected in the adult life of the child. Various classifications are considered, as well as characteristics of the abuser and of the abused.

Key words: child abuse; child abuse, sexual; Mexico
A ctualmente vivimos inmersos en un ambiente lleno de violencia, situación de la que desafortunadamente no escapan los niños. El maltrato infantil (MI) ha llegado a ser un problema que se incrementa en forma alarmante. Aun cuando no se tienen cifras precisas de la magnitud del mismo, se infiere que su presencia es cada vez más evidente. Por esto es necesario difundir el conocimiento sobre este problema en todos los ámbitos, con la finalidad de prevenirlo, identificarlo e iniciar su abordaje terapéutico temprano, evitando de esta forma las consecuencias y los efectos tan intensos que tiene sobre el ser humano.

(I) Profesor, Facultad de Medicina, Universidad Nacional Autónoma de México (UNAM).

(2) Jefe de la Unidad de Investigación Nezahualcóyotl, Facultad de Medicina, UNAM.

Fecha de recibido: 17 de julio de 1997 - Fecha de aprobado: 4 de noviembre de 1997

Solicitud de sobretiros: Dra. Rosalinda Santana Tavira. Av. Valle del Don 104-2,Valle de Aragón 3a. sección, 55280 Ecatepec, Estado de México. 
El MI es un fenómeno que surge con el hombre, por lo que es tan antiguo como la humanidad. ${ }^{1,2}$ También es un problema universal, ${ }^{3} \mathrm{y}$ al respecto Manterola afirma: "El maltrato a los niños no es un mal de la opulencia ni de la carencia, sino una enfermedad de la sociedad". ${ }^{4}$

El MI se ha convertido en un conflicto al que actualmente se enfrentan las diferentes disciplinas implicadas en su abordaje, puesto que no se presenta en forma aislada sino que involucra una gran variedad de factores biopsicosociales. ${ }^{5,6}$

Durante siglos la agresión al menor ha sido justificada de diversas formas; se les ha sacrificado para agradar a los dioses o mejorar la especie, ${ }^{1,7}$ o bien como una forma de imponer disciplina. ${ }^{1,3,7-11}$

En la historia encontramos mitos, leyendas y descripciones literarias referentes a la actitud de exterminio y maltrato hacia los menores. En la mitología se relata que Saturno devora a su progenie y que Medea mata a sus dos hijos para vengarse de Jasón. En la Biblia se relata el caso de Abraham, quien estuvo a punto de sacrificar a su hijo Isaac, así como la matanza de los inocentes ordenada por Herodes. ${ }^{1,7}$ En la historia, 400 años a.C., Aristóteles decía: "Un hijo o un esclavo son propiedad, y nada de lo que se hace con la propiedad es injusto" ${ }^{1,12}$ En el siglo IV d.C., en la antigua Grecia, las niñas eran sacrificadas, en tanto que en Jericó los niños eran empotrados en los cimientos de las murallas, muros de los edificios y puentes, para supuestamente fortalecerlos. ${ }^{13}$ El Códice Mendocino describe diversos tipos de castigos que se imponían a los menores como pincharlos con púas de maguey, hacerlos aspirar humo de chile quemado, dejarlos sin comer, quemarles el pelo, largas jornadas de trabajo, etcétera. ${ }^{1}$ Asimismo, un rey de Suecia llamado Aun sacrificó a nueve de sus 10 hijos con el afán de prolongar su vida. El infanticidio también fue una forma de eliminar a los niños con defectos físicos; durante el nazismo se ordenaba matarlos con el fin de alcanzar la supuesta pureza de la raza, y en algunos países como China, se usaba para controlar la natalidad. ${ }^{1,13}$

Margaret Lynch ${ }^{13}$ describe casos de maltrato mencionados por el médico griego Soranus en el siglo II, en tanto que otros fueron identificados en los siglos XVII, XVIII y XIX; por ejemplo, el de la pequeña Mary Ellen (1874), quien era cruelmente golpeada y encadenada por sus padres adoptivos. Ante esta situación sus vecinos decidieron llevarla a los tribunales, pero tuvo que ser representada por la Sociedad Protectora de Animales al no existir instancia legal alguna que la defendiera. A raíz de este suceso surge en Nueva York la primera Sociedad para la Prevención de la Crueldad en los Niños, y posteriormente se crearon sociedades semejantes en varios países, ${ }^{7,12}$ no obstante, el síndrome del niño golpeado fue descrito por primera vez en 1868 por Ambrosie Tardieu, catedrático de medicina legal en París, luego de realizar las autopsias de 32 niños golpeados y quemados. ${ }^{1,14}$ Posteriormente, en 1946 Caffey describió la presencia de hematomas subdurales asociados con alteraciones radiológicas de los huesos largos en los pequeños. ${ }^{1,7,12,14}$ Henry Kempe y Silverman, en 1962, crearon la expresión síndrome del niño golpeado, con base en las características clínicas presentadas por los casos que ingresaban al servicio de pediatría del Hospital General de Denver, en Colorado. ${ }^{15,16}$ Este concepto fue ampliado por Fontana al indicar que estos niños podían ser agredidos no sólo en forma física sino también emocionalmente o por negligencia, de modo que sustituyó el término golpeado por el de maltratado; ${ }^{7}$ desde entonces se ha publicado una serie de artículos sobre el concepto, que trata de abarcar las diferentes modalidades que existen en cuanto a la acción de lesionar a un niño, mismas que se describen a continuación.

\section{Definición y clasificación}

Es primordial establecer una definición adecuada del MI para elaborar un diagnóstico y un tratamiento apropiados, pues además es el fundamento para establecer un marco legal, proceso que no es fácil dada la complejidad del problema. ${ }^{17-19}$

La definición se ha modificado y enriquecido con las aportaciones de los investigadores que se han abocado al tema, a partir de la primera emitida por Kempe en 1962, quien originalmente define el MI como el uso de la fuerza física no accidental, dirigida a herir o lesionar a un niño, por parte de sus padres o parientes. ${ }^{15}$ Posteriormente se incluyen la negligencia y los aspectos psicológicos como partes del MI; ;,12 para Wolfe es "la presencia de una lesión no accidental, resultado de actos de perpetración (agresión física) o de omisión (falta de atención por parte de quienes están a cargo del niño y que requiere de atención médica o intervención legal)" ${ }^{17}$ La Comisión Nacional de Derechos Humanos $(\mathrm{CNDH})$ propone la siguiente definición: "Todo acto u omisión encaminado a hacer daño aun sin esta intención pero que perjudique el desarrollo normal del menor" ${ }^{20}$ El Sistema Nacional para el Desarrollo Integral de la Familia (DIF) define a los niños maltratados como: “Los menores de edad que enfrentan y sufren ocasional o habitualmente, violencia física, emocional o ambas, ejecutadas por actos de acción u omisión, pero siempre en forma intencional, no accidental, por padres, tutores, custodios o personas responsables de ellos". ${ }^{21}$ Kieran y $\mathrm{O}^{\prime}$ Hagan hacen una 
diferenciación entre maltrato emocional y psicológico; en relación con el primero indican: "Es la respuesta emocional inapropiada, repetitiva y sostenida a la expresión de emoción del niño y su conducta acompañante, siendo causa de dolor emocional (p.e., miedo, humillación, ansiedad, desesperación, etc.) lo cual inhibe la espontaneidad de sentimientos positivos y adecuados, ocasionando deterioro de la habilidad para percibir, comprender, regular, modular, experimentar y expresar apropiadamente las emociones produciendo efectos adversos graves en su desarrollo y vida social".

El maltrato psicológico en cambio es "la conducta sostenida, repetitiva, persistente e inapropiada (violencia doméstica, insultos, actitud impredecible, mentiras, decepciones, explotación, maltrato sexual, negligencia y otras) que daña o reduce sustancialmente tanto el potencial creativo como el desarrollo de facultades y procesos mentales del niño (inteligencia, memoria, reconocimiento, percepción, atención, imaginación y moral) que lo imposibilita a entender y manejar su medio ambiente, lo confunde y/o atemoriza haciéndolo más vulnerable e inseguro afectando adversamente su educación, bienestar general y vida social". ${ }^{22}$

En relación con la clasificación del MI, los investigadores que se dedican al estudio de este tema proponen varias formas de clasificación, mismas que se encuentran en el cuadro I. ${ }^{1,2,18,23,24}$

Consideramos además que las investigaciones sobre el MI abren nuevas líneas -que se precisarán en su momento- en aspectos considerados hoy poco comunes o poco analizados como son: los niños de la frontera y de la guerra, el maltrato étnico, así como el denominado ritualismo satánico, entre otros, donde además de los problemas socioeconómicos subyace un fenómeno transcultural. ${ }^{1,25}$ Otro ejemplo muy claro es el caso del maltrato fetal, que puede ser considerado como un rubro específico e incluido en una nueva clasificación, por la importancia que tiene y el análisis que requiere. . $26-29^{2}$

\section{Etiología}

Los estudios realizados en varios países señalan que el MI es un problema multicausal, en el que intervienen las características del agresor, el agredido, el medio ambiente que les rodea y un estímulo disparador de la agresión. 1,6,7,12,17,30-32

Se han realizado diversas investigaciones con el propósito de determinar el perfil del agresor y de la víctima. $\mathrm{Al}$ respecto se ha encontrado que la figura parental que más agrede es la madre. ${ }^{30-34}$ Se describen algunas características del agresor, tales como: autoestima baja, individuos deprimidos o con tendencia a la depresión, neuróticos, ansiosos, alcohólicos, drogadictos, impulsivos, hostiles, con poca tolerancia a la frustración, con una percepción inadecuada respecto al niño y con antecedentes de maltrato en su niñez. . $-3,12,17,35,36^{-1}$

En relación con las características del menor agredido, frecuentemente se presentan: problemas de salud (congénitos o adquiridos); niños hiperactivos, en su difícil manejo, con bajo rendimiento escolar, y generalmente, hijos no deseados. ${ }^{1,3,12,14,17,30}$

El disparador de la agresión puede ser una mala relación de pareja, problemas económicos, desempleo, vivienda inadecuada, etcétera. $13,3,11,17,30,37-39$

Santos ${ }^{40}$ citando a De Paul y Arruabarrena menciona las características físicas y conductuales de los niños con síndrome de maltrato infantil, así como de sus agresores. En el cuadro II sugerimos los indicadores del agredido y del agresor frente a diferentes tipos de maltrato.

Cuadro I

Clasificación del maltrato INFANTIL SEgún diversos aUtores

\begin{tabular}{|c|c|c|c|c|c|c|c|}
\hline \multirow[t]{2}{*}{ Autor } & \multicolumn{7}{|c|}{$m$} \\
\hline & Físico & Sexual & Emocional & Psicológico & Social & Negligencia & Otros \\
\hline Loredo ' & Agresión física & Abuso sexual & Deprivación emocional & & & & $\begin{array}{l}\text { Formas poco comunes: Síndrome de } \\
\text { Munchausen en niños, abuso fetal, abuso de } \\
\text { tipo ritual o religioso, niños de la guerra y } \\
\text { de la calle }\end{array}$ \\
\hline Martínez ${ }^{2}$ & Físico & Sexual & Deprivación afectiva & & & Descuido & \\
\hline Claussen ${ }^{18}$ & Físico & & & Psicológico & & Negligencia & \\
\hline Suárez ${ }^{23}$ & Físico & & & Psicológico & Social & & \\
\hline Milling 24 & Físico & Abuso sexual & & Psicológico & & Negligencia & \\
\hline
\end{tabular}




\section{Epidemiología}

En su informe anual de 1996 la Organización de las Naciones Unidas para la Infancia (UNICEF, por sus siglas en inglés) establece: en Africa occidental y central se estima que hasta un $20 \%$ de los niños se encontraban desarrollando actividades diversas de tipo laboral; en Africa oriental y meridional se observa que "va en aumento el número de familias cuyo jefe es un niño", y este organismo intensifica estrategias tendientes a la protección especial de niños víctima de negligencia, maltratados, prostituidos y niños de la calle. En Asia oriental y el Pacífico los cambios sociales acontecidos se relacionan con un incremento explosivo de niños que requieren de protección debido a que se ven obligados a trabajar o a irse a las calles. En Asia meridional los niños que trabajan oscilan entre los 35 y 88 millones, muchos de los cuales laboran en situaciones peligrosas y de explotación. En América y el Caribe se observa que en países como Argentina,
Ecuador, México, Uruguay y Venezuela, debido al aumento de las tensiones socioeconómicas y del desempleo y a la disminución del salario, se incrementaron los índices de violencia, a tal grado que en países como Colombia y Guatemala éstos constituyen una de las principales causas de defunción en el grupo de 5 a 14 años de edad. Los disturbios socioeconómicos en Europa central y oriental, en estados independientes y bálticos, se han reflejado en una menor cantidad de matrimonios, incremento de divorcios y aumento de la violencia en el hogar. “Una elevada inflación siguió asolando lospaíses eslavos de la ex Unión Soviética y desarticulando cada vez más la trama social de Belarús, la federación de Rusia y Ucrania. Las mujeres y los niños fueron las principales víctimas del Cáucaso, así como en algunos sectores de las repúblicas de Asia central, donde las instituciones del Estado no pudieron sufragar los costos de los servicios imprescindibles". ${ }^{41}$

En nuestro país no se conoce la prevalencia de niños con MI; los estudios que existen sólo muestran

\section{Cuadro II \\ TIPO DE MALTRATO INFANTIL. INDICADORES DEL NIÑO Y CARACTERÍSTICAS DEL AGRESOR}

\begin{tabular}{|c|c|}
\hline $\begin{array}{l}\text { Tipo de } \\
\text { maltrato }\end{array}$ & $\frac{A g r e}{\text { Indica }}$ \\
\hline Físico & $\begin{array}{l}\text { Huellas del objeto agresor (cinturón, } \\
\text { lazo, zapato, cadena, plancha, etc.) } \\
\text { Eritema, equimosis, inflamación, de- } \\
\text { formación de la región, fractura, ruptura } \\
\text { visceral. Ingresos frecuentes a hospital } \\
\text { por lesiones cuya causa no es clara }\end{array}$ \\
\hline Sexual & $\begin{array}{l}\text { Presencia en genitales y/o ano de: equi- } \\
\text { mosis, laceraciones, sangrado, prurito, } \\
\text { inflamación, himen perforado (niñas), } \\
\text { dificultad para caminar, semen, infec- } \\
\text { ciones, somatización. En adolescentes y } \\
\text { adultas, dispareunia }\end{array}$ \\
\hline
\end{tabular}

Psicológico Retraso psicomotor

\section{Actitudes agresivas, destructivas, re-} beldes, hiperactividad o apatía, timidez, miedo, ansiedad, aislamiento, culpa, sentimiento de ser malos. En el ámbito escolar es frecuente la inasistencia y el bajo rendimiento
Miedo, ansiedad, culpa, desconfianza, enojo.

En adolescentes y adultos: atracción homosexual, anorgasmia, pérdida o disminución de la libido, autodevaluación

Deterioro de facultades mentales, principalmente en el área cognitiva, autodevaluación y bajo rendimiento escolar
Impide amistades con el menor del sexo opuesto. Estimula - excita sexualmente al menor usando revistas, películas o por exhibición

Devalúa constantemente al menor en público o en privado. Lo culpa de todo lo malo que sucede. No se muestra cariñoso. Constantemente amenaza o aterroriza al niño

Es rechazante, poco tolerante, no presta atención, no dedica tiempo al niño relacionarse con los demás, callados, poco expresivos, tímidos, sensación de no ser queridos, de ser rechazados, bloqueo emocional
Negligencia Higiene deficiente, desnutrición en grado variable, aspecto enfermizo, ropa inadecuada para el lugar o clima. Habitación inadecuada. Sin asistencia médica oportuna, astenia y adinamia permanentes
Retraimiento, apatía, depresión, timidez, asistencia irregular a la escuela, bajo rendimiento escolar, indiferencia al medio ambiente externo
Incapaces de controlar sus imulsos, se observan enojados, al más mínimo estímulo res

Apático, desinteresado, ve al menor como una carga
Problemas en la relación de pareja Problemas económicos

Frustración Hostilidad

Alto potencial de agresión

Antecedente de haber sido maltratado

Presencia de algún tipo de adicción

Tienden al aislamiento No se autorreconocen como maltratadores

Incapacidad de buscar ayuda profesional Idea distorsionada del niño

Exigen más de lo que el niño puede hacer

Tiende frecuentemente a la depresión Problemas de adicción: alcohol, drogas 
facetas parciales de este problema, ${ }^{1,6,30,34}$ por lo que es necesario establecer estrategias de investigación adecuadas para su detección. En 1995, la UNICEF estimaba que la población menor de 19 años en México representaba $47 \%$ del total de los habitantes del país y que el grupo de 0 a 4 años correspondía al $25.8 \%$, por lo que su preocupación "son los niños y las niñas que viven en condiciones de extrema pobreza y/o que pertenecen a etnias, principalmente los pequeños menores de cinco años por ser la población infantil más vulnerable a enfermedades infecciosas, a desnutrición, al abuso y al maltrato". ${ }^{42}$

En 1993 la Procuraduría General de Justicia del Distrito Federal informaba que el abuso sexual ocupaba el segundo lugar de los delitos notificados en esta institución; la incidencia era de $24.1 \%$, del cual casi el total $(23.9 \%)$, correspondía a agresiones que habían sido perpetradas hacia menores de 13 años. ${ }^{43}$

Levav y colaboradores ${ }^{11}$ afirman que en 1991, en Estados Unidos de América (EUA), se notificaron 2.7 millones de casos de niños maltratados o desatendidos; citando a Tonella y Zuppinger mencionan que "en Suiza, país que ha pasado siglos enteros sin guerras, un tercio de los padres imponen castigos corporales a sus hijos".

Martínez, citando a Gallayher, refiere: "La frecuencia de maltrato infantil es alta, $18 \%$ de todos los niños están sujetos a alguna forma de maltrato, muchos mueren y otros quedan lisiados de por vida. En EUA 4000 niños mueren al año debido a lesiones causadas por un adulto".

En España, Verdugo ${ }^{44}$ informa que $11.5 \%$ de los niños intelectualmente discapacitados son víctima de diferentes tipos de maltrato y que el más frecuente es la negligencia física.

Teresinha ${ }^{45}$ refiere que "en Brasil la miseria, el hambre, las enfermedades, los malos tratos, el abandono, la prisión ilegal, la tortura física, la permanente amenaza de muerte y el asesinato es a lo que se enfrentan cotidianamente, sobre todo en las últimas décadas, millones de niños, adolescentes y jóvenes en edad escolar que viven en la calle. Todas estas formas de violencia les dificulta o impide frecuentar la escuela". En este país se identifica a los niños de la calle o a aquel que realiza un trabajo informal con el concepto de niño carente, abandonado, sin hogar o pivete, porque necesariamente es un menor que se vale de la violencia para sobrevivir en la ciudad; es el pequeño ladrón, traficante o delincuente, por lo que algunos grupos de empresarios se han vinculado con los "escuadrones de la muerte" para exterminarlos, afirmando que "cuando se mata un pivete se está haciendo un beneficio a la sociedad". De enero de 1987 a julio de
1988 se notificaron 1397 casos de asesinatos de niños, adolescentes y jóvenes, en su mayoría varones de 11 a 18 años de edad, negros y mulatos.

Como podemos observar el problema es universal, y a pesar de ello seguimos desconociendo la prevalencia de esta enfermedad en cada uno de los países.

\section{Repercusiones}

Existe una estrecha relación entre los diferentes tipos de maltrato y el desarrollo biopsicosocial del niño -así lo demuestran los estudios realizados en estas áreas-, lo que puede traducirse en problemas escolares, tanto en el plano cognitivo como en el de la interacción social, ${ }^{46-50}$ y en alteraciones de la conducta manifestadas por agresión y retraimiento. ${ }^{10,49,51}$ Luntz y colaboradores ${ }^{52}$ además relacionan esto con el abuso de sustancias tóxicas, delincuencia, criminalidad y los suicidios. Gracia ${ }^{53}$ establece que existe un desajuste personal y social en estos niños. El abuso sexual se asocia con problemas de somatización, ansiedad, hostilidad, miedo, rechazo, depresión y desconfianza. ${ }^{54-57}$ Valenzuela y colaboradores ${ }^{43}$ refieren en sus investigaciones una mayor frecuencia de problemas psicosomáticos y diversas alteraciones del comportamiento sexual en personas que tienen antecedentes de abuso sexual en la niñez, en tanto que otros autores lo relacionan con trastornos de la personalidad más severos. ${ }^{58}$

Destaca en este aspecto lo desarrollado a partir del análisis del maltrato fetal y su repercusión en el vínculo afectivo que se establece en el binomio madre-hijo desde la etapa preconceptiva. ${ }^{26,29,59-61}$

\section{Tratamiento}

Se requiere de un tratamiento integral no sólo de la víctima sino también de su agresor y de la familia, para lo cual es necesario un equipo multidisciplinario que se encargue de esta problemática y aborde los aspectos biológicos, psicológicos y legales pertinentes, por lo que se necesita de la planeación y la coordinación de estos servicios y de personal profesional capacitado. ${ }^{6,62-64}$ La terapia familiar está dirigida primero a reconocer el problema, establecer una adecuada comunicación entre los integrantes de la misma, modificar los patrones conductuales de los padres eliminando las tácticas punitivas o coercitivas y sustituyéndolas por métodos positivos más efectivos. ${ }^{62}$ Wolfe ${ }^{17}$ propone un plan de intervención y prevención cuya meta sea restablecer la integridad, así como el funcionamiento efectivo intrafamiliar, y cuyos objetivos sean: a) adquirir destrezas en la educación de los niños con el fin de manejar problemas típicos y difíciles relacio- 
nados con ellos; b) promover el uso de métodos de control positivos hasta eliminar el castigo; c) desarrollar estrategias para solucionar problemas en situaciones críticas y que sean operativas para la familia; d) regular las respuestas violentas y el comportamiento impulsivo que lesionan a la familia, y e) promover la interacción social de los miembros de la misma, reduciendo su aislamiento físico y psicológico del resto de la comunidad.

Foncerrada ${ }^{30}$ señala que cuando la psicodinamia familiar está muy alterada y no existen vínculos afectivos o están muy deteriorados, lo más conveniente es que el menor no regrese a su hogar por el riesgo de sufrir una nueva agresión; pero cuando existen vínculos afectivos que en el momento se encuentran debilitados, lo ideal es tratar de preservar la integridad familiar proporcionando apoyo a la familia mediante la colaboración de otro miembro de la misma capaz de modelar los cuidados parentales; promover visitas domiciliarias por parte de personal especializado que actúe como "conciencia moral"; favorecer el contacto físico entre padre, madre e hijo; mejorar el apego afectivo, y orientar a los padres sobre las características conductuales del niño según su etapa de desarrollo. Todo lo anterior aumenta la confianza y los recursos del agresor para responder a las necesidades del niño, así como su capacidad de control para enfrentar los problemas. La combinación de la terapia individual, conyugal y de grupo contribuye a generar autoaceptación, impulsando hacia el cambio.

Por su parte Maher ${ }^{13}$ considera que no sólo es necesario prestar atención al menor, la familia y las circunstancias que los rodean, sino también al equipo multidisciplinario profesional, su funcionamiento y su problemática, con el fin de que el sistema trabaje eficazmente y ofrezca protección infantil "sin causar el mínimo daño a la familia"; por este motivo, según Maher debe tomarse en cuenta el compromiso, preparación profesional en el manejo del MI, la cooperación, comprensión y conocimiento profundo de la dinámica familiar.

Consideramos que en nuestro país se requiere de una mayor interacción de los organismos encargados de la protección del menor maltratado, así como destinar más recursos financieros y humanos (equipo multidisciplinario capacitado) para abordar el problema en forma integral y proporcionar alternativas de solución cada vez de mayor calidad. ${ }^{62}$ Como Bertolli ${ }^{65}$ señala, es necesario continuar con las diferentes líneas de investigación a fin de establecer las estrategias metodológicas más adecuadas para conocer la realidad de este problema.

\section{Conclusiones}

Las raíces históricas, culturales y sociales constituyen un reto a vencer al enfrentar el MI. ${ }^{25}$ Las diversas definiciones y clasificaciones de este fenómeno muestran las dificultades para abordarlo principalmente en dos terrenos: primero, las distintas facetas que el problema posee, y segundo, sus implicaciones éticas y legales. Llama la atención el surgimiento de aspectos y perspectivas distintas que presentan el problema de la clasificación como un proceso en un momento de gran inmadurez. Es fundamental reunir a los investigadores y expertos del área para trabajar en este aspecto particular, principalmente en torno a lo psicológico y emocional; una alternativa sería la formación de un grupo de consenso respecto al MI en nuestro país. En cuanto a la etiología, profundizar en las características del agresor, agredido, disparador de la agresión y de su interacción permitirá establecer estrategias preventivas que impidan maltratar al menor.

Pensamos que en el terreno de la epidemiología la investigación tiene un reto preponderante; una vez superados los aspectos históricos y culturales, será posible contar con definiciones claras y precisas que nos permitan obtener un registro confiable y una visión más real del problema.

El estudio de las repercusiones abre otro panorama que ofrece aclarar aspectos del desarrollo de la personalidad y de las alteraciones de la conducta en el adulto, quien hoy puede generar un niño agredido, y mañana, otro agresor. Esta es otra motivación importante para continuar con el desarrollo de la investigación en el área, lo que irá de la mano con la exploración de distintas alternativas para su manejo y ofrecer resultados más congruentes con la realidad.

Proponemos un cuadro que tendrá que modificarse con experiencias posteriores; describe diversos aspectos del maltrato que deben ser urgentemente abordados. Asimismo, constituye una herramienta eje para el diagnóstico (cuadro II).

Siendo el MI un fenómeno multicausal y universal, consideramos que la divulgación, instrucción y educación de la población en general sobre el mismo son estrategias prioritarias que pueden permitir hacer conciencia a todos; es necesario dar a conocer que no sólo el trauma físico lesiona a un niño, sino que también los otros tipos de maltrato, como el emocional, el psicológico y la negligencia, deterioran la personalidad del pequeño, y de aquí la importancia de identificar cada uno de estos indicadores. Coincidimos con otros investigadores citados en que el abordaje terapéutico es integral e incluye a todo un equipo especializado en 
la solución de esta problemática. También es necesario precisar desde dónde un acto puede ser considerado como maltrato, es decir, unificar criterios en cuanto a la definición y la clasificación del MI, así como promover y realizar investigaciones en nuestra población, con nuestros niños, debido a que son pocas las que se hacen en este terreno, entre las que destaca el trabajo que dirige el doctor Loredo Abdalá, quien desde hace muchos años se ha preocupado por los diversos aspectos del maltrato. Se requiere, como lo menciona Osorio, ${ }^{12}$ de recursos económicos tendientes a defender $\mathrm{y}$ proteger en forma real a los pequeños.

Recuérdese que: "El niño maltratado de hoy es el adulto conflictivo de mañana".

\section{Agradecimientos}

Expresamos nuestro más sincero agradecimiento a los doctores: Víctor Manuel Mendoza Núñez, por su valiosa asesoría, motivación, y por compartir su experiencia en el campo de la investigación; José Luis Esquinca, por su asesoría; Miguel Ángel Fernández Ortega, Ulises A. Guzmán García, Arnulfo Irigoyen Coria y Jaime Bárcenas Trejo, por su apoyo en la realización de este trabajo.

\section{Referencias}

I. Loredo AA. Maltrato al menor. México D.F.: Interamericana McGrawHill, 1994.

2. Martínez MM, Reyes RR. Maltrato infantil. Un problema inquietante. Bol Med Hosp Infant Mex 1993;50:557-563.

3.Torregosa FL. El niño maltratado. En: El maltrato a los niños y sus repercusiones educativas. Memorias del 2do. Simposio Interdisciplinario e Internacional. México, D.F.: Federación Iberoamericana contra el Maltrato Infantil, 1992;1I:55-74.

4. Manterola MA. La prevención del maltrato a los menores en México. En: El Maltrato a los niños y sus repercusiones educativas. Memorias del 2 do. Simposio Interdisciplinario e Internacional. México, D.F.: Federación Iberoamericana contra el Maltrato Infantil, I992; I: 173-184.

5. Santamaría GR. El maltrato infantil o maltrato social. En: El Maltrato a los niños y sus repercusiones educativas. Memorias del 2 do. Simposio Interdisciplinario e Internacional. México, D.F.: Federación Iberoamericana contra el Maltrato Infantil, 1992,II:20I-206.

6. Loredo AA, Oldak SD, Carbajal RL, Reynes MJ.Algoritmo del niño maltratado a su ingreso a un centro hospitalario pediátrico. Bol Med Hosp Infant Mex 1990;47:91-95.

7. Fontana V. En defensa del niño maltratado. México, D.F.: Editorial Pax, 1979.

8. Forward S. Las deidades parentales. Padres que odian. México, D.F.: Grijalbo, 1991.

9. Corral VV, Frías AM, Romero M, Muñoz A. Validity of a scale measuring beliefs regarding the "positive" effects of punishing children: A study of punishing children: A study of Mexican mothers. Child Abuse Negl 1995; 19:669-679.
10. Muller RT, Hunter EJ, Stollak G. The intergenerational transmission of corporal punishment: A comparison of social learning and temperament models. Child Abuse Negl 1995; 19:1323-1335.

II. Levav I, Guerrero R, Phebo L, Coe G, Cerqueira MT. El castigo corporal en la niñez: ¿Endemia o epidemia? Bol Oficina Sanit Panam 1996; 120:229-239.

12. Osorio CA. El niño maltratado. 2a. edición. México, D.F.:Trillas, 1995.

13. Maher P. El abuso contra los niños. La perspectiva de los educadores. México, D.F.: Grijalbo, 1990.

14. Kempe RS, Kempe HC. Niños maltratados. 3a. edición. Madrid: Morata, 1985.

15. Kempe HC, Silverman NF, Steele BF, Droegemueller W, Silver HK. The battered-child syndrome. JAMA 1962;181:105-1/2.

16. Kempe HC. Paediatric implications of the battered baby syndrome. Arch Dis Child 197I;46:28-37.

17. Wolfe D. Programa de conducción de niños maltratados. México, D.F.: Trillas, 1991.

18. Claussen AH, Crittenden PM. Physical and psychological maltreatment: Relations among types of maltreatment. Child Abuse Negl 1991;15:5-18. 19. Gough D. Defining the problem. Child Abuse Negl 1996;20:993-1002. 20.Aguilar AM. Maltrato a menores. En: El maltrato a los niños y sus repercusiones educativas. Memorias del 2do. Simposio Interdisciplinario e Internacional. México. D.F.: Federación Iberoamericana contra el Maltrato Infantil, 1992:192-200.

21. Gamboa CF. Prevención al maltrato del menor y su maltrato social. En: El maltrato a los niños y sus repercusiones educativas. Memorias del 2 do. Simposio Interdisciplinario e Internacional. México, D.F.: Federación Iberoamericana contra el Maltrato Infantil, 1992:300-307.

22. Kieran PO. Emotional and psychological abuse: Problems of definition. Child Abuse Negl 1995; 19:446-46।.

23. Suárez Y.El maltrato y sus repercusiones en la educación. En: El maltrato a los niños y sus repercusiones educativas. Memorias del $2 \mathrm{do}$. Simposio Interdisciplinario e Internacional. México, D.F.: Federación Iberoamericana contra el Maltrato Infantil, 1992:323-336.

24. Milling-Kinard E. Methodological issues and practical problems in conducting research on maltreated children. Child Abuse Negl 1994;18:645656.

25. Barreto PL, Suárez OE. Maltrato de niños y adolescentes: una perspectiva internacional. Bol Oficina Sanit Panam 1996;121:123-137.

26. Kent L, Laidlaw JDD, Brockington IF. Fetal abuse. Child Abuse Negl 1997;21:181-186.

27. Morey MA, Begleiter ML, Harris DJ. Profile of a battered fetus. Lancet 1981;2: 1294-1295.

28. Stafford PA, Biddinger PW, Zumwalt RE. Lethal intrauterine fetal trauma.Am J Obstet Gynecol 1988;159:485-489.

29. Tyler R, Howard J, Espinosa M, Simpson DS. Placement with substanceabusing mothers vs placement with other relatives: Infant outcomes. Child Abuse Negl 1997;21:337-349.

30. Foncerrada-Moreno M. El niño víctima de maltrato. Rev Med Inst Mex Seguro Soc 1982;20:457-469.

3I. Ulate-Mora F, Madrigal-Tellini S. Maltrato a menores en una comunidad costarricense. Bol Med Hosp Infant Mex 199I;48:355-360.

32. Loredo-Abdalá A, Reynes-Manzur J, De Martínez CS, Carbajal-Rodríguez L, Vidales-Bayona C, Villaseñor-Zepeda J. El maltrato al menor. Una realidad creciente en México. Bol Med Hosp Infant Mex 1986;43: 425-434.

33. Loredo AA. El maltrato del niño: algunas consideraciones éticas sobre su atención. Bol Med Hosp Infant Mex 1993;50:898-902.

34. Herrada-Huidobro A, Nazar-Beutelspacher A, Cassaball-Núñez M,VegaRamos R, Nava-Cruz C. El niño maltratado en Tlaxcala: estudio de casos. Salud Publica Mex 1992;34:626-634.

35. Lesnik-Obertein M, Arend JK, Cohen Leo. Parental hostility and its sources in psychologically abusive mothers:A test of the three-factor theory. Child Abuse Negl 1995; 19:33-49. 
36. Merrill LL, Herving KL, Milner SJ. Childhood parenting experiences, intimate partner conflict resolution and adult risk for child physical abuse. Child Abuse Negl 1996;20:1049-1065.

37. Jones DE, McCurdy K. The links between types of maltreatment and demographic characteristics of children. Child Abuse Negl 1992;16: 20I215 .

38. Krishan V, Morrison BK. An ecological model of child maltreatment in Canadian province. Child Abuse Negl 1995; 19:101-1 13.

39. Drake B, Pandey S. Understanding the relationship between neighborhood poverty and specific types of child maltreatment. Child Abuse Negl 1996;20:1003-1018.

40. Santos DD. Intervención de la policía Vasca en el maltrato infantil. En: El Maltrato a los niños y sus repercusiones educativas. Memorias del 2 do. Simposio Interdisciplinario e Internacional. México, D.F.: Federación Iberoamericana contra el Maltrato Infantil, | 992:3 | I-322.

4I. Gorita I. Informe anual. Fondo de las Naciones Unidas para la Infancia. México, D.F.: UNICEF, 1996:4-36.

42. Cuentas ZJC. Fondo de las Naciones Unidas para la Infancia en México. Primero la niñez. México, D.F.: UNICEF, 1996:3-31.

43.Valenzuela J, Hernández GL, Sánchez SJJ. Quejas psicosomáticas y comportamiento de adolescentes en función de abuso sexual en la niñez.Arch Hispanoam Sexol 1995; I:20I-224.

44. Verdugo MA, Bermejo BG. The maltreatment of intellectually handicapped children and adolescents. Child Abuse Negl 1995;190:205-215.

45. Teresinha BG. La muerte trágica y violenta impide a niños y jóvenes de la calle frecuentar la escuela en Brasil. En: Memorias del 2do. Simposio Interdisciplinario e Internacional. El maltrato a los niños y sus repercusiones educativas. México, D.F.: Federación Iberoamericana contra el Maltrato Infantil, 1992:154-165.

46. Barnett D,Vondra IJ, Shonk MS. Self-perceptions, motivation and school functioning of low-income maltreated and comparison children. Child Abuse Negl 1996;20:395-410

47. De Paul J,Arruabarrena I. Behavior problems in school-aged, physically abused and neglected children in Spain. Child Abuse Negl 1995; 19:409418

48. Feldman R, Salzinger S, Alvarado L. Parent, teacher and peer rating of physically abused and nonmaltreated children's behavior. J Abnorm Child Psychol 1995;23:317-334.

49. Levendosky AA, Okun A, Parker GJ. Depression and maltreatment as predictors of social competence and social problem-solving skills in schoolage children. Child Abuse Negl 1995; 19:445-452.

50. Kurtz PD, Gaudin MJ, Wodarski SJ, Howing TP. Maltreatment and the school-aged child: School performance consequences. Child Abuse Negl 1993; 17:58|-589.
51. Prino CT, Peyrot M. The effect of child physical abuse and neglect on agressive, withdrawn, and prosocial behavior. Child Abuse Negl |994; |8:87|-884.

52. Luntz BK, Spatz WC. Antisocial personality disorder in abused and neglected children grown up. Am J Psychiatry 1994;151:670-674.

53. Gracia E.Visible but unreported:A case for the "not serious enough" cases of child maltreatment. Child Abuse Negl 1995;19:1083-1093.

54.Whiffen VE, Benazo RN. Discriminant validity of the TSC-40 in an outpatient setting. Child Abuse Negl 1997;21:107-II5.

55. López F, Carpintero E, Hernández A, Martín MJ, Fuertes A. Prevalencia y consecuencias del abuso sexual al menor en España. Child Abuse Negl 1995; 19:1039-1050.

56. Sanders PK, Moisan PA, Wadlington S, Morgan S, English K. Ethnic differences in psychological functioning among black and latin sexually abused girls. Child Abuse Negl 1995; 19:69|-706.

57. Sanders B, Lausen EB. The measurement of psychological maltreatment: Early data on the child abuse and trauma scale. Child Abuse Negl 1995; 19:315-323.

58. Irwin HJ.Traumatic childhood events percieved availability of emotional support and the development of dissociative tendencies. Child Abuse Negl 1996;20:701-707.

59.Vives RJ, Lartigue BT,Arnaldo L, López DA, Padrón AL, Zapata M.Vicisitudes en la estructuración del vínculo maternoinfantil durante la gestación. Cuaderno Psicoanal 1991;XXIV:167-191.

60. Vives RJ, Lartigue BT, Córdoba A.Apego y vínculo. Comunicación preliminar. Cuaderno Psicoanal 1992;XXV:45-53.

6I. Vives RJ, Lartigue BT. Apego y vínculo materno-infantil. México, D.F: Universidad de Guadalajara, 1994:13-94.

62. Santana TR, Irigoyen CA, Herrera BE, Sánchez AR, Mazón RJJ, Fernández OMA. Síndrome de Kempe: pautas de diagnóstico para el médico general y familiar. Rev Fac Med UNAM 1997;40:187-190.

63. Sundell K. Child-care personnel's failure to report child maltreatment: Some Swedish evidence. Child Abuse Negl 1997;21:93-105.

64. Oldfield D, Hays JB, Erickson MM. Evaluation of the effectiveness of project trust: An elementary school-based victimization prevention estrategy. Child Abuse Negl 1996;20:82I-832.

65. Bertolli J, Morgensten H, Sorenson SB. Estimating the ocurrence of child maltreatment and risk-factor effects: Benefits of a mixed-design strategy in epidemiologic research. Child Abuse Negl 1995; 19:1007-1016. 